\title{
Cyclooxygenases and prostaglandin E synthases in the endometrium of the rhesus monkey during the menstrual cycle
}

\author{
Tong Sun ${ }^{1}$, Shi-Jie $\mathrm{Li}^{1}{ }^{\prime}$, Hong-Lu Diao ${ }^{1}$, Chun-Bo Teng ${ }^{1}$, Hong-Bin Wang ${ }^{2}$ \\ and Zeng-Ming Yang ${ }^{1}$ \\ Colleges of ${ }^{1}$ Life Sciences and ${ }^{2}$ Animal Medicine, Northeast Agricultural University, Harbin 150030, China
}

Correspondence should be addressed to Zeng-Ming Yang; Email: zmyang@mail.neau.edu.cn

\begin{abstract}
Cyclooxygenase (COX), a rate-limiting enzyme that produces prostaglandins (PGs) from arachidonic acid, exists in two isoforms, COX-1 and COX-2. PGE 2 synthase (PGES) is a terminal prostanoid synthase and can enzymatically convert the cyclooxygenase product $\mathrm{PGH}_{2}$ to $\mathrm{PGE}_{2}$, including two isoforms: microsomal PGES (mPGES) and cytosolic PGES (cPGES). cPGES is predominantly linked with COX-1 to promote the immediate response. mPGES is preferentially coupled with the inducible COX-2 to promote delayed $\mathrm{PGE}_{2}$ generation. COX-2-deficient female mice are infertile with abnormalities in ovulation, fertilization, implantation and decidualization. The aim of this study was to examine immunohistochemically the expression pattern of COX-1, COX-2, mPGES and CPGES proteins in the endometrium of the rhesus monkey during the menstrual cycle. COX-1 immunostaining was mainly localized in the luminal epithelium and glandular epithelium near the lumen, and detected in all the stages during the menstrual cycle. COX-2 immunostaining was mainly localized in the luminal and glandular epithelium, and strongly shown during the mid-luteal phase (days 16 and 20) of the menstrual cycle. There was a strong cPGES immunostaining in the luminal and glandular epithelium on days 12, 16, 20 and 25 of the menstrual cycle. mPGES immunostaining was strongly detected in the glandular epithelium on days 20 and 25 of the menstrual cycle. These data suggest that the coupling of CPGES and COX-1 in the luminal epithelium may be responsible for the synthesis of $\mathrm{PGE}_{2}$ in monkey endometrium, and the coupling of mPGES and COX-2 in the glandular epithelium may be of importance for preparing the receptive endometrium. Reproduction (2004) 127 465-473
\end{abstract}

\section{Introduction}

Prostaglandins (PGs) are considered to be proinflammatory and to play important roles during female reproduction (Downie et al. 1974, Lim et al. 1997). Cyclooxygenase (COX) is a rate-limiting enzyme that produces PGs from arachidonic acid. There are two isoforms: COX-1 and COX-2. COX-1 appears to be constitutively synthesized in many tissues, although its level of expression can vary with the state of differentiation or following cytokine or tumor promoter stimulation (Seibert et al. 1994, Samet et al. 1995). However, COX-2 expression is upregulated in response to various proinflammatory or mitogenic agents (Hla \& Neilson 1992, Smith et al. 1994). COX function in mice has been demonstrated with gene knockout experiments. COX-1-deficient females are fertile with specific parturition defects, whereas COX-2-deficient females are infertile with abnormalities in ovulation, fertilization, implantation and decidualization (Dinchuk et al. 1995, Langenbach et al. 1995, Lim et al. 1997). Recently, in another study, COX-2-deficient mice did not show any abnormality in embryo implantation except for the reduction in the numbers of ovulated and fertilized eggs, and a delay in decidual growth (Cheng \& Stewart 2003). What causes this difference is still not clear.

$\mathrm{PGE}_{2}$ synthase (PGES) is a terminal prostanoid synthase that can enzymatically convert the cyclooxygenase product $\mathrm{PGH}_{2}$ to $\mathrm{PGE}_{2}$. There are two isoforms of PGES, microsomal PGES (mPGES) and cytosolic PGES (cPGES). cPGES is constitutively expressed in a wide variety of cells and tissues, and predominantly linked with COX-1 to promote the immediate response, during which relatively high concentrations of arachidonic acid are released in a short period (Tanioka et al. 2000). mPGES, a membrane-associated and inducible perinuclear enzyme with glutathione-dependent activity, is expressed in a variety of tissues, including prostate, testes, and small intestine (Jakobsson et al. 1999). mPGES is preferentially coupled with the inducible COX-2 to promote delayed $\mathrm{PGE}_{2}$ generation and, if COX-2 already exists in cells, it also regulates immediate $\mathrm{PGE}_{2}$ generation (Murakami et al. 2000).

The expression and regulation of the mPGES gene in mouse uterus was recently characterized ( $\mathrm{Ni}$ et al. 2002). The strong mPGES expression in the implantation site and 
decidual cells suggests that mPGES might play an important role during implantation and, more importantly, decidualization (Ni et al. 2002). Recently, it has been found that COX-2 mRNA and protein and mPGES mRNA are differentially expressed and regulated in bovine endometrium during the estrous cycle. The COX-2 and mPGES pathway is responsible for the endometrial production of PGE2 in the bovine endometrium during the estrous cycle (Arosh et al. 2002). Expression of mPGE synthase was localized in glandular epithelial and endothelial cells in both basalis and functionalis regions of the human endometrium. By contrast, stromal staining was predominantly localized in the functionalis layer (Milne et al. 2001).

The expression of CPGES in the uterus was reported only in mice. There was a strong level of cPGES mRNA signal in the stromal cells at the implantation site on day 5 of pregnancy, while cPGES immunostaining was strongly detected in the luminal epithelium. The signals for both cPGES mRNA and immunostaining were strongly detected in the decidualized cells from days 6-8 of pregnancy. These data suggest that CPGES may play an important role during implantation and decidualization (Ni et al. 2003).

In spite of the extensive studies reported on COX expression, regulation and potential function in the reproductive processes of other species, relatively little is known on COX expression and regulation in the primate uterus. Kim et al. (1999) have reported the expression of COX-1 and COX-2 during the menstrual cycle and pregnancy in the baboon. In man, COX-2 was localized in the uterine glandular epithelium by immunohistochemistry throughout the menstrual cycle and in early pregnancy (Jones et al. 1997). However, COX expression during the menstrual cycle is still not defined in monkey. The aim of this study was to examine immunohistochemically the expression of COX-1, COX-2, mPGES and cPGES proteins in monkey endometrium during the menstrual cycle.

\section{Materials and Methods}

\section{Animals}

Rhesus monkeys (Macaca mulatta) at the Fujian Province Non-Human Primate Research Center in China were caged individually in a controlled environment with a 12-h light-12-h dark cycle. All animal procedures were approved by the Institutional Animal Care and Use Committee of Northeast Agricultural University (China). Animals were evaluated daily by visual examination of the perineum for menses, with the onset of menses defined as day 1 of the menstrual cycle. Adult female monkeys with regular menstrual cycles of approximately 28 days were chosen for this study. The uterine samples of the menstrual cycle were taken on days 6,9 and 12 of the proliferative phase, and on days 16, 20 and 25 of the luteal phase. All of the monkeys were killed by an overdose injection of ketamine. At least three monkeys were used in each group. The uteri were removed and treated as described below.

\section{Immunohistochemistry}

The dorsal portion of monkey uterus was immediately cut into small pieces, fixed in Bouin's solution for $24 \mathrm{~h}$, dehydrated with graded ethanol and embedded in paraffin. Paraffin sections were cut, deparaffinized and hydrated gradually in PBS. For the sections used for COX-2 immunohistostaining, antigen retrieval was performed in $0.1 \mathrm{mM}$ EDTA $\left(\mathrm{pH}\right.$ 9.2) at $88^{\circ} \mathrm{C}$ water bath for $10 \mathrm{~min}$. Nonspecific binding was blocked with $10 \%$ horse serum in PBS at $37^{\circ} \mathrm{C}$ for $1 \mathrm{~h}$. The sections were incubated with goat anti-COX-1 immunoglobulin G (IgG) (1:100, Santa Cruz Biotechnology, Santa Cruz, CA, USA) or goat antiCOX-2 IgG (1:300, Santa Cruz) at $24^{\circ} \mathrm{C}$ for 90 min. Normal goat $\lg \mathrm{G}$ was used to replace each primary antibody for a negative control. The sections were then incubated with biotinylated rabbit anti-goat antibody followed by an avidin-alkaline phosphatase complex and Vector Red according to the manufacturer's protocol (Vectastain ABCAP kit, Vector Laboratories, Burlingame, CA, USA). For the mPGES and cPGES immunostaining, the sections were incubated with rabbit anti-mPGES (1:200, Cayman Chemical, Ann Arbor, MI, USA) or rabbit anti-cPGES (1:400, Cayman Chemical) overnight at $4{ }^{\circ} \mathrm{C}$. The sections were then incubated with biotinylated goat anti-rabbit antibody followed by an avidin-alkaline phosphatase complex and Vector Red. Vector Red was visualized as a red color. Endogenous alkaline phosphatase activity was inhibited with levamisole (Sigma). The sections were counterstained with hematoxylin and mounted.

Additionally, normal rabbit IgG was used to replace the primary antibodies for a negative control. Moreover, rabbit anti-CPGES was preincubated with the blocking peptide for CPGES (Cat no. 360150, Cayman Chemical) for $30 \mathrm{~min}$ prior to incubating with the sections on day 25 of the menstrual cycle. Similarly, the rabbit anti-mPGES antibody was preincubated with mPGES blocking peptide (Cat No. 360140, Cayman Chemical) for 30 min prior to incubating with the sections on day 25 of the menstrual cycle.

The degree of staining was assessed subjectively by blinded examination of the slides by two investigators. A scoring system was designated as $0=$ no stain, $1=$ pale stain, $2=$ moderate stain, $3=$ strong stain and $4=$ very intense stain. At least three monkeys at each stage were used under this analysis. The means \pm S.E.M. of the staining intensity for each cellular compartment were shown graphically.

\section{Results \\ COX-1 immunostaining}

COX-1 immunostaining in monkey endometrium during the menstrual cycle is shown in Fig. 1 and scored in Fig. 2. COX-1 immunostaining was detected in the luminal 

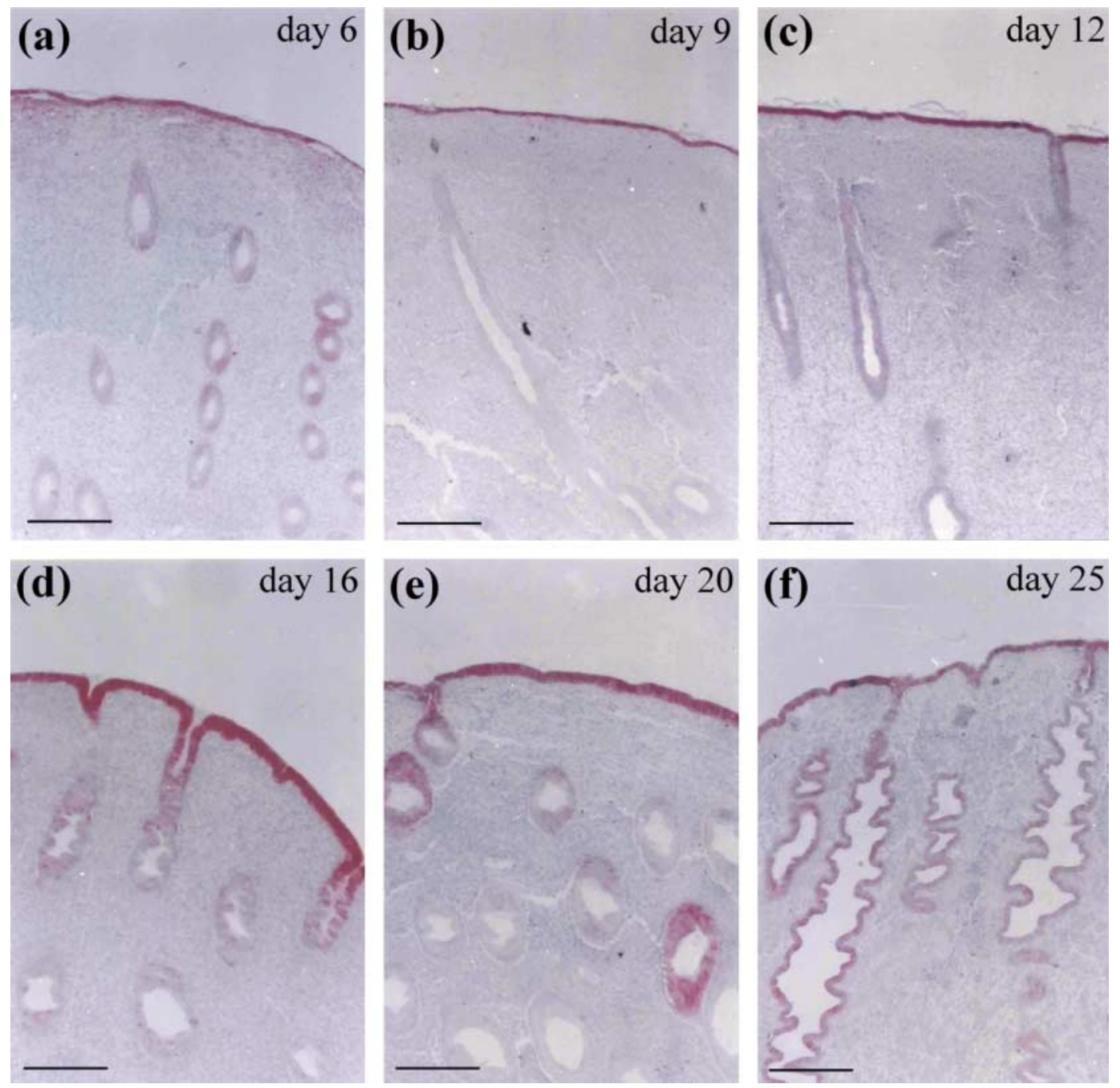

Figure 1 COX-1 immunostaining in monkey endometrium on days 6 (a), 9 (b), 12 (c), 16 (d), 20 (e) and 25 (f) of the menstrual cycle. Scale bars represent $30 \mu \mathrm{m}$.



Figure 2 COX-1 immunostaining intensity in monkey endometrium during the menstrual cycle. Open bars, luminal eithelium; black bars, glands in functionalis; hatched bars, glands in basalis; gray bars, stroma (no bar, staining was undetectable in that part of the tissue). Each bar represents the mean \pm S.E.M. of the immunostaining intensity. 
epithelium throughout the menstrual cycle (Fig. 1). However, a high level of COX-1 staining was seen on days 12, 16 and 20 of the menstrual cycle, being at the highest level on day 16 (Fig. 1c-e). The level of COX-1 staining in the glandular epithelium was different between basalis and functionalis regions of the endometrium. No immunostaining was detected in the glandular epithelium of the basalis region, while a low level of COX-1 staining was detected in the glandular epithelium of the functionalis region except for day 9 of the menstrual cycle (Fig. 1b). There was no detectable COX-1 immunostaining in the stroma (Fig. 1). Additionally, there was no detectable COX-1 immunostaining when goat anti-COX-1 IgG was replaced by normal goat IgG (data not shown).

\section{COX-2 immunostaining}

COX-2 immunostaining in monkey endometrium during the menstrual cycle is shown in Fig. 3 and scored in Fig. 4.
There was no detectable COX-2 immunostaining in the endometrium on days 6 and 9 (Fig. 3a and b). A basal level of COX-2 immunostaining was seen in the luminal epithelium and glands in the functionalis region on day 12 (Fig. 3c). COX-2 immunostaining was strongly observed in the luminal epithelium and glandular epithelium of the functionalis region on day 16 (Fig. 3d). There was a low level of COX-2 immunostaining in the luminal epithelium and glandular epithelium of the functionalis region on day 20 (Fig. 3e). A basal level of COX-2 immunostaining was seen in the luminal epithelium and glandular epithelium of the functionalis region on day 25 (Fig. 3f). During the whole menstrual cycle, there was no detectable COX-2 immunostaining in the glandular epithelium of basalis regions or in the stroma (Fig. 3a-f). Additionally, there was no detectable COX-2 immunostaining when goat anti-COX-2 IgG was replaced by normal goat IgG (data not shown).
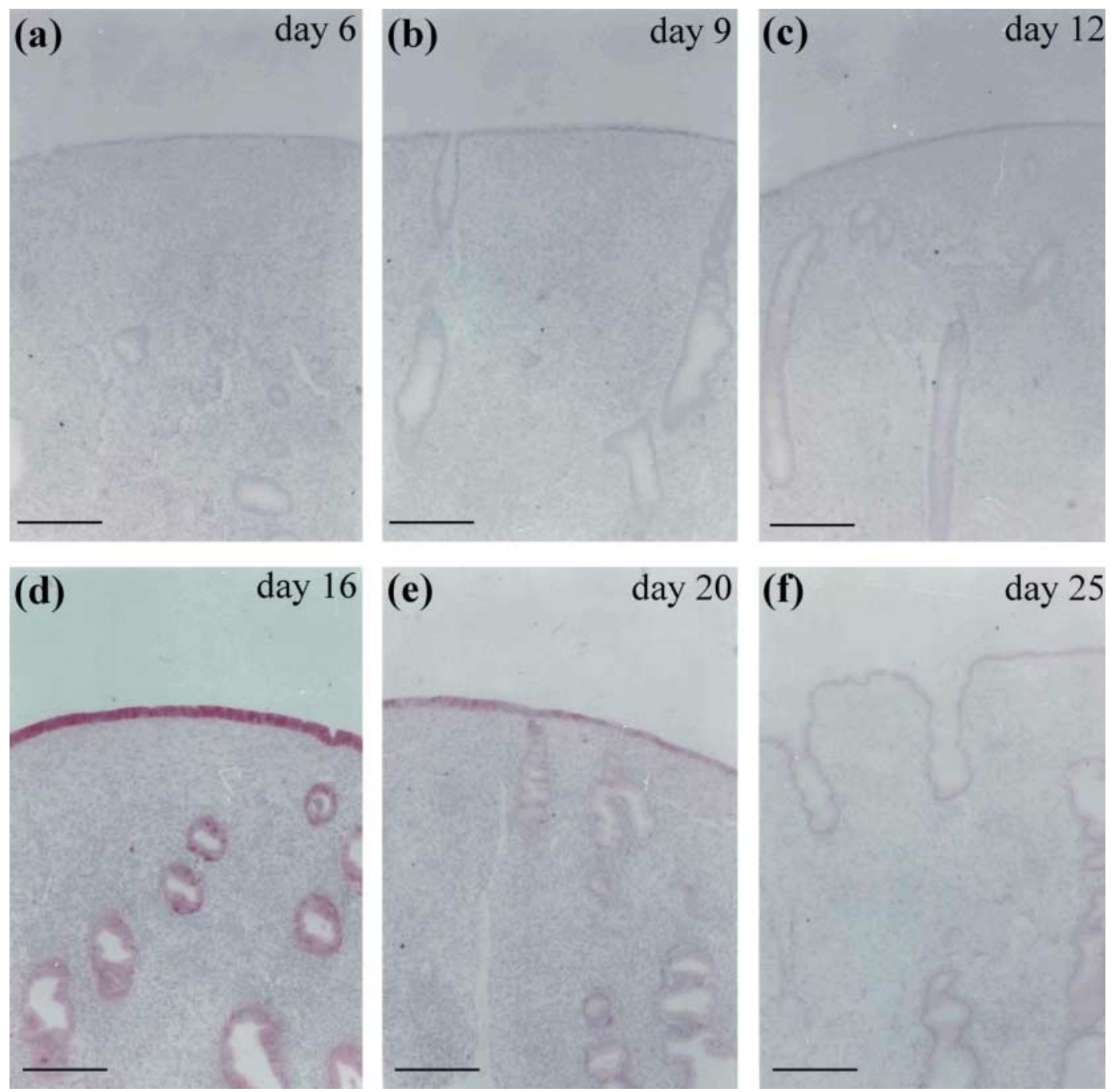

Figure 3 COX-2 immunostaining in monkey endometrium on days 6 (a), 9 (b), 12 (c), 16 (d), 20 (e) and 25 (f) of the menstrual cycle. Scale bars represent $30 \mu \mathrm{m}$. 




Figure 4 COX-2 immunostaining intensity in monkey endometrium during the menstrual cycle. Open bars, luminal epithelium; black bars, glands in functionalis; hatched bars, glands in basalis; gray bars, stroma (no bar, staining was undetectable in that part of the tissue). Each bar represents the mean \pm S.E.M. of the immunostaining intensity.
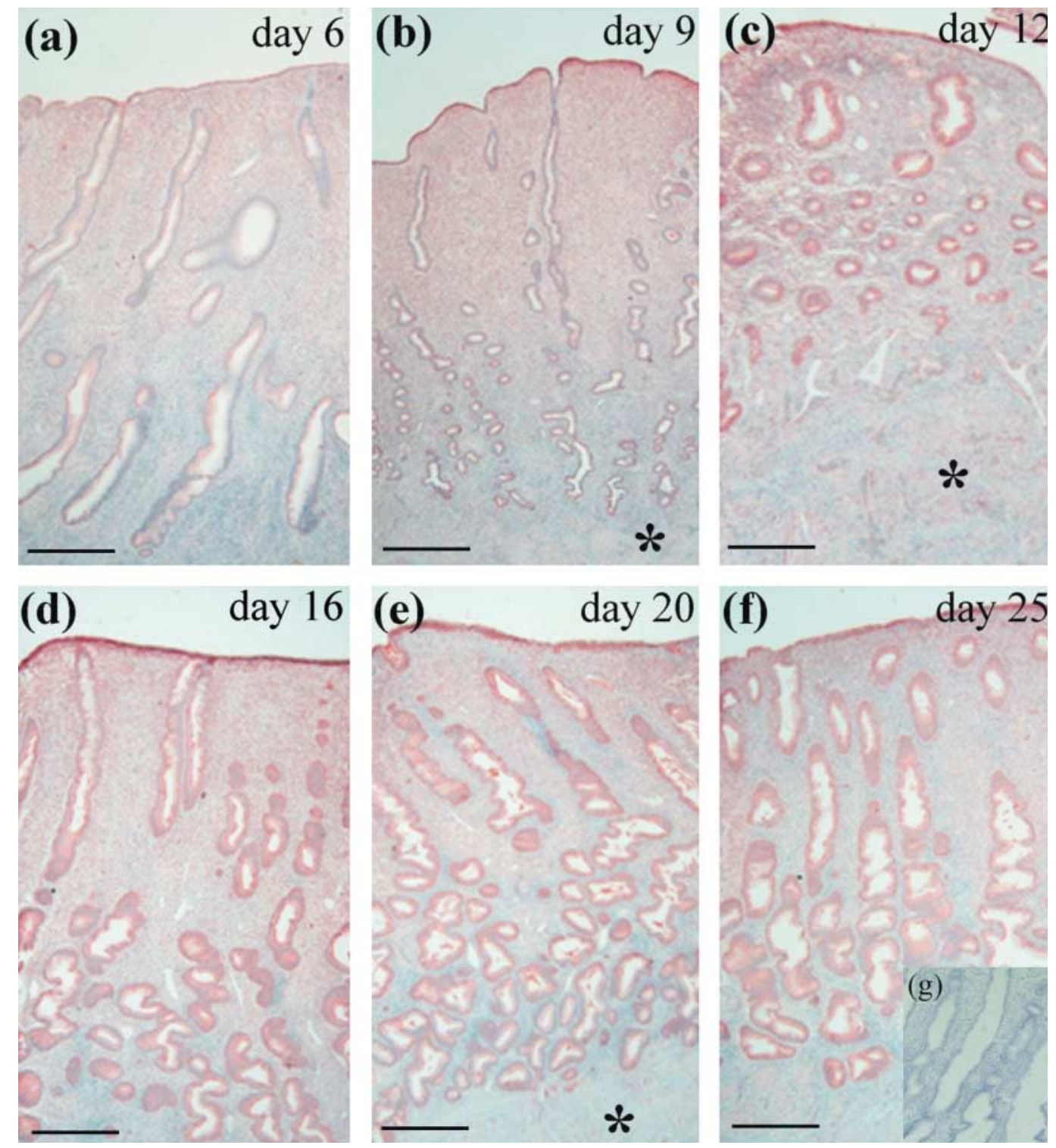

Figure 5 cPGES immunostaining in monkey endometrium on days 6 (a), 9 (b), 12 (c), 16 (d), 20 (e) and 25 (f) of the menstrual cycle. (g) There was no detectable cPGES immunostaining in the endometrium on day 25 of the menstrual cycle when the rabbit anti-cPGES antibody was preincubated with cPGES blocking peptide. *Myometrium. Scale bars represent $35 \mu \mathrm{m}$. 


\section{cPGES immunostaining}

CPGES immunostaining in monkey endometrium during the menstrual cycle is shown in Fig. 5 and scored in Fig. 6. On day 6 , a basal level of cPGES immunostaining was observed only in the luminal and glandular epithelium (Fig. 5a). On day 9, a low level of cPGES immunostaining was seen in the glandular epithelium of the basalis region and in the luminal epithelium, while only a basal level was observed in the glandular epithelium of the functionalis region and the entire stroma (Fig. $5 b$ ). On days 12, 16, 20 and 25, cPGES immunostaining was highly detected in the luminal epithelium, stroma and glandular epithelium located in both basalis and functionalis regions (Fig. 5c-f). However, when the rabbit anti-cPGES antibody was preincubated with cPGES blocking peptide, there was no detectable cPGES immunostaining in the endometrium on day 25 of the menstrual cycle (Fig. $5 \mathrm{~g}$ (inset)).

\section{mPGES immunostaining}

MPGES immunostaining in monkey endometrium during the menstrual cycle is shown in Fig. 7 and scored in Fig. 8. There was a low level of mPGES immunostaining only in the luminal epithelium on day 6 (Fig. 7a). No immunostaining was seen in the luminal and glandular epithelium of functionalis region on days 9,12 and 16 (Fig. 7b, $d$ and e), while a basal level of mPGES immunostaining was seen in the glandular epithelium of the basalis region on days 9, 12 and 16 (Fig. 7c-e). No immunostaining was seen in the luminal epithelium on days 20 and 25 (Fig. $7 f$ and $h$ ). On day 20, a low level of mPGES immunostaining was seen in the glandular epithelium of the functionalis region (Fig. $7 \mathrm{f}$ ), while a high level in the glandular epithelium of the basalis region (Fig. $7 \mathrm{~g}$ ). On day 25, a high level of mPGES immunostaining was detected in the whole glandular epithelium (Fig. 7h and i). However, there was no detectable mPGES immunostaining in the glandular epithelium on day 25 of the menstrual cycle when the rabbit anti-mPGES antibody was preincubated with mPGES blocking peptide (Fig. 7j (inset)).

\section{Discussion}

In this study, COX-1 immunostaining was mainly detected in the luminal epithelium and glandular epithelium near the lumen, the same as COX-1 localization in mouse, baboon, mink and human endometrium (Chakraborty et al. 1996, Song et al. 1998, Kim et al. 1999, Marions \& Danielsson 1999). However, in ovine endometrium, COX1 immunostaining was localized in both epithelial and stromal cells (Charpigny et al. 1997). COX-2 immunostaining was localized in the luminal and glandular epithelium in this study, like that in the western spotted skunk and sheep (Charpigny et al. 1997, Das et al. 1999). In human endometrium, COX-2 immunostaining was mainly seen in the human luminal epithelium and perivascular cells (Marions \& Danielsson 1999). COX-2 was localized in the luminal epithelium and stroma in mouse, mink and baboon endometrium (Chakraborty et al. 1996, Kim et al. 1999). These findings suggest that the localization of COX-1 and COX-2 in the endometrium is slightly different among various species.

It has been shown that CPGES is predominantly linked with COX-1 to promote the immediate response, during which relatively high concentrations of arachidonic acid are released in a short period (Tanioka et al. 2000). In our study, CPGES was colocalized with COX-1 in the luminal epithelium and glandular epithelium, and both cPGES and COX-1 were highly expressed on days 16 and 20 of the menstrual cycle. In addition, culture studies have demonstrated that both human epithelial and stromal cells can synthesize $\mathrm{PGE}_{2}$ in vitro (Smith \& Kelly 1988). These data suggest that the coupling of CPGES and COX-1 in the luminal epithelium may be responsible for the synthesis of $\mathrm{PGE}_{2}$ in monkey endometrium.

In this study, mPGES immunostaining was mainly detected in the glandular epithelium on days 20 and 25 of



Figure 6 CPGES immunostaining intensity in monkey endometrium during the menstrual cycle. Open bars, luminal epithelium; black bars, glands in functionalis; hatched bars, glands in basalis; gray bars, stroma (no bar, staining was undetectable in that part of the tissue). Each bar represents the mean \pm S.E.M. of the immunostaining intensity. 



Figure 7 mPGES immunostaining in monkey endometrium on days 6 (a), 9 (b and c), $12(d), 16(e), 20$ ( $f$ and g) and 25 (h and i) of the menstrual cycle. (j) There was no detectable mPGES immunostaining in the glandular epithelium on day 25 of the menstrual cycle when the rabbit antimPGES antibody was preincubated with mPGES blocking peptide. *Myometrium. Scale bars represent $25 \mu \mathrm{m}$.

the menstrual cycle. In human endometrium, mPGES immunoreactivity in the functionalis layer was expressed in glandular epithelial, stromal, and endothelial cells (Milne et al. 2001). mPGES is preferentially coupled with the inducible COX-2 to promote delayed $\mathrm{PGE}_{2}$ generation and, if COX-2 already exists in cells, it also regulates immediate $\mathrm{PGE}_{2}$ generation (Jakobsson et al. 1999, Murakami et al. 2000). In the present study, COX-2 


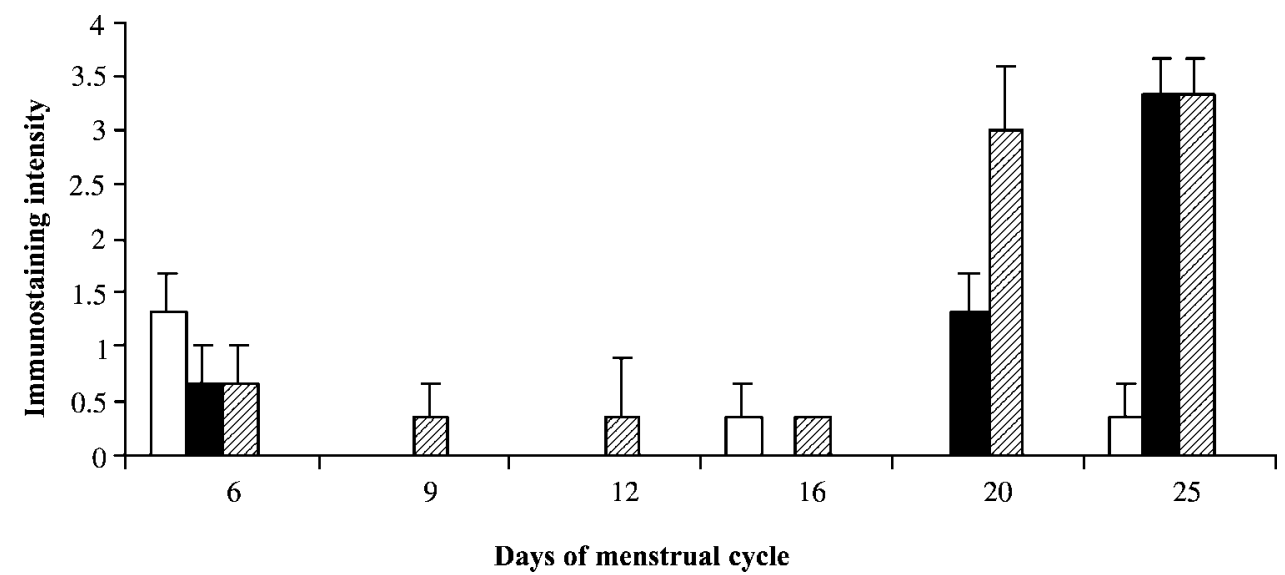

Figure 8 mPGES immunostaining intensity in monkey endometrium during the menstrual cycle. Open bars, luminal epithelium; black bars, glands in functionalis; hatched bars, glands in basalis; gray bars, stroma (no bar, staining was undetectable in that part of the tissue). Each bar represents the mean \pm S.E.M. of the immunostaining intensity.

immunostaining was strongly detected in the luminal epithelium and glandular epithelium on day 16, and weakly on day 20. However, mPGES immunostaining was strongly seen only in the glandular epithelium on days 20 and 25. The expression peak of COX-2 was not matched with that of mPGES. COX-2 was colocated with mPGES in the glandular epithelium only on day 20 of the menstrual cycle, which was within the potential implantation window. Nevertheless, it is important to see whether COX-2 and $\mathrm{mPGES}$ are highly coexpressed in the endometrium of rhesus monkey during the peri-implantation period. During early pregnancy in mice, both COX-2 and mPGES were highly expressed at implantation sites on day 5 of pregnancy (Chakraborty et al. 1996, Ni et al. 2002). Furthermore, COX-2-deficient mice had multiple failures in female reproductive processes, including ovulation, fertilization, implantation and decidualization (Lim et al. 1997). Conversely, Cheng and Stewart (2003) reported that COX-2-deficient mice did not show any abnormalities in embryo implantation except for the reduction in the numbers of ovulated and fertilized eggs, and a delay in decidual growth. This difference may be caused by the different genetic backgrounds of the COX-2-deficient mice used by the two groups. The role of COX-2 during embryo implantation also needs to be further investigated.

The exact role of $\mathrm{PGE}_{2}$ in the monkey endometrium is not fully elucidated. It seems that COX-1 and CPGES are mainly responsible for the synthesis of $\mathrm{PGE}_{2}$ in the monkey endometrium during the menstrual cycle. In bovine endometrium, COX-1 mRNA and protein are not expressed on any day of the estrous cycle, whereas COX-2 mRNA, and protein and mPGES mRNA are differentially expressed and regulated in bovine endometrium during the estrous cycle. The COX-2 and mPGES pathway is responsible for the endometrial production of $\mathrm{PGE}_{2}$ in the bovine endometrium during the estrous cycle (Arosh et al. 2002). In human colon epithelial cells, overexpression of COX-2 and enhanced synthesis of $\mathrm{PGE}_{2}$ have been shown to promote the proliferation and survival of cells through inhibition of apoptosis. $\mathrm{PGE}_{2}$ has been shown to upregulate expression of antiapoptotic genes such as bcl-2 (Sheng et al. 1998). It is plausible that, in the human endometrium, $\mathrm{PGE}_{2}$ may be activating similar mechanisms that promote glandular epithelial cell proliferation and/or survival (Milne et al. 2001). $\mathrm{PGE}_{2}$ function in glandular epithelial cells may also be associated with the regulation of uterine angiogenesis. In the human endometrium, vascular endothelial growth factor expression is localized to the glandular epithelial cells throughout the menstrual cycle (Torry et al. 1996). Moreover, $\mathrm{PGE}_{2}$ has been previously shown to upregulate the expression of vascular endothelial growth factor in a number of different cell types (Harada et al. 1994, Cheng et al. 1998). Nevertheless, the availability of arachidonic acid may be a rate-limiting step in synthesizing $\mathrm{PGE}_{2}$. Cytosolic phospholipase $\mathrm{A}_{2 \alpha}\left(\mathrm{CPLA}_{2 \alpha}\right)$ is a major provider of arachidonic acid in the COX system for PG biosynthesis. $\mathrm{CPLA}_{2 \alpha}$ deficiency results in aberrant uterine spacing of embryos and deferred implantation (Song et al. 2002). It remains to determine whether arachidonic acid is provided for $\mathrm{PGE}_{2}$ synthesis through the COX-1/cPGES and/or COX-2/mPGES pathway.

In conclusion, our data suggest that the coupling of CPGES and COX-1 in the luminal epithelium may be responsible for the synthesis of $\mathrm{PGE}_{2}$ in monkey endometrium, and the coupling of mPGES and COX-2 in the glandular epithelium may be important in preparing the receptive endometrium.

\section{Acknowledgements}

This work was supported by Chinese National Natural Science Foundation grants 39825120, 30330060 and 30270163, and the Special Fund for Major State Basic Research Project (G1999055903). This work was also funded by CICCR Program subproject CIG-01-64 of the CONRAD Program, Eastern Virginia Medical School, Virginia, USA. The views expressed by the authors do not necessarily reflect the views of CONRAD or CICCR. 


\section{References}

Arosh JA, Parent J, Chapdelaine P, Sirois J \& Fortier MA 2002 Expression of cyclooxygenases 1 and 2 and prostaglandin $\mathrm{E}$ synthase in bovine endometrial tissue during the estrous cycle. Biology of Reproduction 67 161-169.

Chakraborty I, Das SK, Wang J \& Dey SK 1996 Developmental expression of the cyclo-oxygenase- 1 and cyclo-oxygenase-2 genes in the peri-implantation mouse uterus and their differential regulation by the blastocyst and ovarian steroids. Journal of Molecular Endocrinology 16 107-122.

Charpigny G, Reinaud P, Tamby JP, Creminon C, Martal J, Maclouf J \& Guillomot M 1997 Expression of cyclooxygenase-1 and -2 in ovine endometrium during the estrous cycle and early pregnancy. Endocrinology 138 2163-2171.

Cheng JG \& Stewart CL 2003 Loss of cyclooxygenase-2 retards decidual growth but does not inhibit embryo implantation or development to term. Biology of Reproduction 68 401-404.

Cheng T, Cao W, Wen R, Steinberg RH \& LaVail MM 1998 Prostaglandin $E_{2}$ induces vascular endothelial growth factor and basic fibroblast growth factor mRNA expression in cultured rat Muller cells. Investigative Ophthalmology and Visual Science $\mathbf{3 9}$ 581-591.

Das SK, Wang J, Dey SK \& Mead RA 1999 Spatiotemporal expression of cyclooxygenase 1 and cyclooxygenase 2 during delayed implantation and the periimplantation period in the western spotted skunk. Biology of Reproduction 60 893-899.

Dinchuk JE, Car BD, Focht RJ, Johnston JJ, Jaffee BD, Covington MB, Contel NR, Eng VM, Collins RJ, Czerniak PM, Gorry SA \& Trzaskos JM 1995 Renal abnormalities and an altered inflammatory response in mice lacking cyclooxygenase II. Nature $\mathbf{3 7 8}$ 406-409.

Downie J, Poyser NL \& Wunderlich M 1974 Levels of prostaglandins in human endometrium during the normal menstrual cycle. Journal of Physiology 236 465-472.

Harada S, Nagy JA, Sullivan KA, Thomas KA, Endo N, Rodan GA \& Rodan SB 1994 Induction of vascular endothelial growth factor expression by prostaglandin $E_{2}$ and $E_{1}$ in osteoblasts. Journal of Clinical Investigation 93 2490-2496.

Hla T \& Neilson K 1992 Human cyclooxygenase-2 cDNA. PNAS 89 7384-7388.

Jakobsson PJ, Thoren S, Morgenstern R \& Samuelsson B 1999 Identification of human prostaglandin $\mathrm{E}$ synthase: a microsomal, gluthathione-dependent, inducible enzyme, constituting a potential novel drug target. PNAS 96 7220-7225.

Jones RL, Kelly RW \& Critchley HOD 1997 Chemokine and cyclooxygenase-2 expression in human endometrium coincides with leukocyte accumulation. Human Reproduction 12 1300-1306.

Kim JJ, Wang J, Bambra C, Das SK, Dey SK \& Fazleabas AT 1999 Expression of cyclooxygenase- 1 and -2 in the baboon endometrium during the menstrual cycle and pregnancy. Endocrinology $1402672-2678$.

Langenbach R, Morham SG, Tiano HF, Loftin CD, Ghanayem BI, Chulada PC, Mahler JF, Lee CA, Goulding EH, Kluckman KD, Kim HS \& Smithies O 1995 Prostaglandin synthase I gene disruption in mice reduces arachidonic acid-induced inflammation and indomethacin-induced gastric ulceration. Cell 83 483-492.

Lim H, Paria BC, Das SK, Dinchuk JE, Langenbach R, Trzaskos JM \& Dey SK 1997 Multiple female reproductive failures in cyclooxygenase 2-deficient mice. Cell 91 197-208.
Marions L \& Danielsson KG 1999 Expression of cyclo-oxygenase in human endometrium during the implantation period. Molecular Human Reproduction 5 961-965.

Milne SA, Perchick GB, Boody SC \& Jabbour HN 2001 Expression, localization, and signaling of $\mathrm{PGE}_{2}$ and EP2/EP4 receptors in human nonpregnant endometrium across the menstrual cycle. Journal of Clinical Endocrinology and Metabolism $\mathbf{8 6}$ 4453-4459.

Murakami M, Naraba $H$, Tanioka T, Semmyo N, Nakatani $Y$, Kojima F, Ikeda T, Fueki M, Ueno A, Ohishi S \& Kudo I 2000 Regulation of prostaglandin $\mathrm{E}_{2}$ biosynthesis by inducible membrane-associated prostaglandin $E_{2}$ synthase that acts in concert with cyclooxygenase-2. Journal of Biological Chemistry 275 32783-32792.

Ni H, Sun T, Ding NZ, Ma XH \& Yang ZM 2002 Differential expression of microsomal PGE synthase at the implantation sites and in the decidual cells in mouse uterus. Biology of Reproduction $67351-358$.

Ni H, Sun T, Ma XH \& Yang ZM 2003 Expression and regulation of cytosolic prostaglandin $\mathrm{E}$ synthase in mouse uterus during the periimplantation period. Biology of Reproduction 68 744-750.

Samet JM, Fasano MB, Fonteh AN \& Chilton FH 1995 Selective induction of prostaglandin $\mathrm{G} / \mathrm{H}$ synthase I by stem cell factor and dexamethasone in mast cells. Journal of Biological Chemistry 270 8044-8049.

Seibert K, Zhang Y, Leahy K, Hauser S, Masferrer J, Perkins W, Lee L \& Isakson P 1994 Pharmacological and biochemical demonstration of the role of cyclooxygenase 2 in inflammation and pain. PNAS 91 12013-12017.

Sheng H, Shao J, Morrow JD, Beauchamp RD \& DuBois RN 1998 Modulation of apoptosis and bcl-2 expression by prostaglandin $\mathrm{E}_{2}$ in human colon cancer cells. Cancer Research 58 362-366.

Smith SK \& Kelly RW 1988 The release of $\mathrm{PGF}_{2 \alpha}$ and $\mathrm{PGE}_{2}$ from separated cells of human endometrium and decidua. Prostaglandins, Leukotrienes and Essential Fatty Acids 33 91-96.

Smith WL, Meade EA \& DeWitt DL 1994 Interactions of PGH synthase isozymes- 1 and -2 with NSAIDs. Annals of the New York Academy of Science 744 50-57.

Song JH, Sirois J, Houde A \& Murphy BD 1998 Cloning, developmental expression, and immunohistochemistry of cyclooxygenase 2 in the endometrium during embryo implantation and gestation in the mink (Mustela vison). Endocrinology 139 3629-3636.

Song H, Lim H, Paria BC, Matsumoto H, Swift LL, Morrow J, Bonventre JV \& Dey SK 2002 Cytosolic phospholipase $A_{2 \alpha}$ is crucial for 'on-time' embryo implantation that directs subsequent development. Development 129 2879-2889.

Tanioka T, Nakatani Y, Semmyo N, Murakami M \& Kudo I 2000 Molecular identification of cytosolic prostaglandin $E_{2}$ synthase that is functionally coupled with cyclooxygenase- 1 in immediate prostaglandin $E_{2}$ biosynthesis. Journal of Biological Chemistry 275 32775-32782.

Torry DS, Holt VJ, Keenan JA, Harris G, Caudle MR \& Torry RJ 1996 Vascular endothelial growth factor expression in cycling human endometrium. Fertility and Sterility $6672-80$.

Received 14 February 2003

First decision 15 April 2003

Revised manuscript received 7 January 2004

Accepted 15 January 2004 